\title{
A Multi-site Real-time Co-simulation Platform for the Testing of Control Strategies of Distributed Storage and V2G in Distribution Networks
}

\author{
E. Bompard*, A. Monti ${ }^{+}$, A. Tenconi*, \\ A. Estebsari", T. Huang ${ }^{*}$, E. Pons ${ }^{*}$, M. Stevic ${ }^{+}$, S. Vaschetto ${ }^{*}$, S. Vogel ${ }^{+}$ \\ *POLITECNICO DI TORINO \\ C.so Duca degli Abruzzi, 24, 10129 \\ Torino, Italy \\ Tel.: +39 / (011) - 0907148 \\ E-Mail: ettore.bompard@polito.it, alberto.tenconi@polito.it \\ URL: http://www.polito.it \\ ${ }^{+}$ACS, E.ON ENERGY RESEARCH CENTER, RWTH AACHEN UNIVERSITY \\ Mathieustraße 10, 52074 \\ Aachen, Germany \\ Tel.: +49 / (0241) - 8049700 \\ E-Mail: AMonti@eonerc.rwth-aachen.de \\ URL: http://www.acs.eonerc.rwth-aachen.de/
}

\section{Keywords}

«Smart grids», «Distribution of electrical energy», «Energy system management», «Energy storage», «Vehicle to Grid», «Real-time co-simulation».

\begin{abstract}
This paper presents a real-time co-simulation platform aimed to test control strategies for the management of the interaction between a smart grid and active prosumers. The main feature of the proposed framework relies on the multi-site approach that allows the decoupling between the network model and the system under test. This allows separate testing with the exchange of a limited amount of information between the two systems, helping to preserve the confidentiality of data belonging to different parties. As an example the paper addresses the development and testing of a distributed storage and vehicle-to-grid management system connected to a real distribution network model.
\end{abstract}

\section{Introduction}

The growing environmental awareness, together with the need to reduce the dependence on fossil resources, has led to a widespread diffusion of Renewable Energy Sources (RES), such as photovoltaic and wind. Power systems were designed for delivering the electrical power produced in a few big centralized power plants to the final passive users, thanks to a structure constituted by generation, transmission and distribution systems. Now, due to the penetration of RES, the paradigm is shifting towards an active distribution network with a multitude of small-scale distributed generators and prosumers [1], [2].

A large deployment of distributed storage systems connected to the network could be used to overcome the time mismatch between energy demand and production from RES that are usually nonprogrammable and scarcely predictable. A step towards this objective can be achieved resorting to the Vehicle-to-Grid (V2G) concept that consists on the introduction of smart strategies and technologies for the interaction between Plug-in EVs (PEVs) and the power grid [3], [4]. In particular, the PEVs onboard rechargeable batteries are exploited as distributed storage systems. From the grid point of view, V2G can represent a distributed source of stored energy for ancillary services (those services needed to keep the network sustainable). V2G may also be used to supply power during the on-peak periods, discharging the battery, and contributing to smoothing the power profile and increasing the load factor [1]. In this perspective, from the network point of view, V2G changes the paradigm of the electric vehicles from loads only, to active prosumers. The basic scenario for the V2G exploitation is 
characterized by the deployment of technologies that allow transferring electricity and information from the power grid to the vehicle and vice-versa. The bilateral communication between the EV and the distribution grid allows matching, at best and in real time, the need of both, given their technological constraints [1].

For these reasons, power systems are becoming more and more complex and there is the need for considering, modelling and studying the complex interactions between the prosumers and the networks. In this scenario, the smart management of both the network and the prosumers assumes a fundamental role, with a smartness massively embedded into grids assets, consisting in decentralized capability of the grid components of sensing, computing and communicating [5].

In this paper the management of different configurations of distributed storage and V2G systems connected to a distribution network is analyzed, with the main goal of local energy balance. Local energy balancing means trying to reduce the energy dependence of a portion of distribution system from the transmission network, by minimizing the power flow through the HV/MV transformers, or by keeping it in a desired range.

The proposed investigation is performed using a multi-site real-time co-simulation platform. The realtime simulation can be fruitfully applied to the study of smart grids as it provides the possibility of testing ex-ante the control strategies and the devices on simulated networks, before applying them in the real grids [6], [7]. Besides, multi-site co-simulation allows for expanding capabilities of individual laboratories in order to enable the study of large-scale and interdisciplinary problems. Recently there has been a significant increase in interest in the concept of multi-site co-simulation, transferring data over the Internet. Feasibility, applicability and benefits of a real-time co-simulation over Internet for joint operation in a single experiment were demonstrated in [8] and [9]. Multi-site co-simulation also enables technical cooperation between different parties that is often challenged by confidentiality aspects, allowing simulation and testing with the exchange of a limited amount of information between the decoupled models [10], [11].

Thus, to exploit the benefits of the multi-site co-simulation, the proposed investigation is based on a subsystem representing the model of a real distribution network, and a subsystem representing the distributed storage and V2G prosumers with their own management system. Through a limited number of information, exchanged using a Virtual Private Network (VPN), the two subsystems are simulated in real-time on two different Opal-RT platforms located at RWTH Aachen (Germany) and Politecnico di Torino (Italy), respectively.

This paper is organized with a brief description of the proposed multi-site co-simulation platform for decoupled testing. Then, the case study based on both distributed storage and V2G connected to a real distribution network model is described and, finally, simulation results for the case study are reported.

\section{A multi-site co-simulation platform for decoupled testing}

The multi-site real-time co-simulation is performed by interconnecting multiple real-time simulators located in different laboratories that can even be placed at different geographic locations. Each realtime simulator exchanges data through User Datagram Protocol (UDP) to a Sim2Sim server. In this application UDP is better than Transmission Control Protocol (TCP): in fact in real-time communication it is preferable to discard reordered and lost packets instead of waiting for a packet retransmission. The different Sim2Sim servers manage the multipoint connection through a VPN, establishing the connection between remote labs. The Sim2Sim servers forward data between simulators, drop invalid packets and collect statistics on the communication link. The chosen VPN is based on the open source software Tinc [12] which is running on the Sim2Sim servers.

In this paper the problem is decoupled in two subsystems. The first is the model of the distribution network, while the second one is the model of distributed storage and V2G with their management system. The two subsystems are modelled respectively at RWTH Aachen and at Politecnico di Torino on two Opal-RT real time targets (Fig. 1).

Thanks to the decoupling of the problem in two separate subsystems, managing the interconnection through the Internet and exchanging a limited amount of data and information, it is possible to test different software or hardware components, developed by different parties, without disclosing confidential information. In this paper we show how the management system of the V2G and storage devices can be tested and validated in Torino (Italy), using a distribution network model running in Aachen (Germany). 


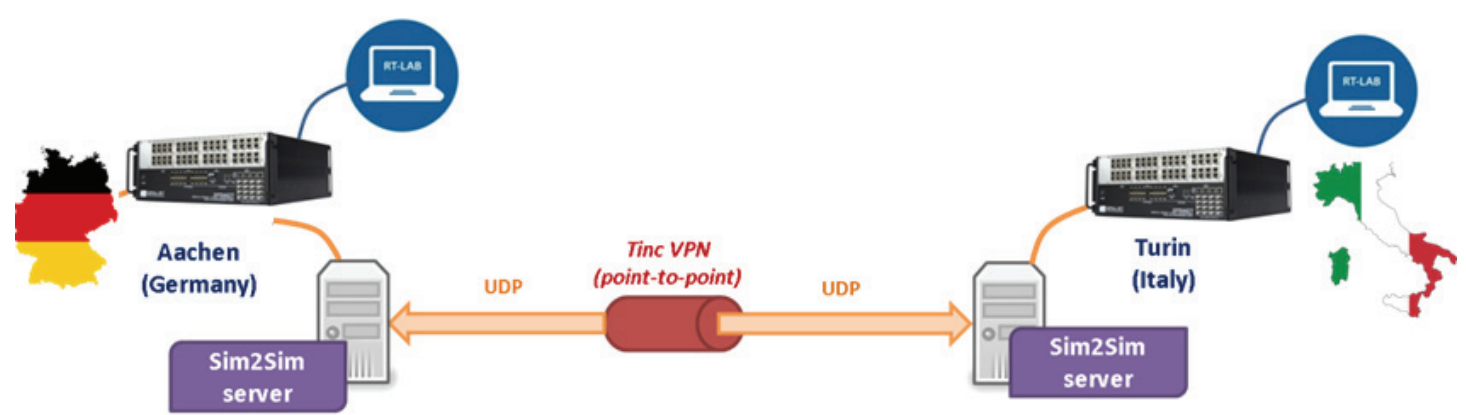

Fig.1. Scheme of the interconnection for the real-time co-simulation.

\section{Case study}

The case study presented in this paper consists in testing a management strategy for distributed storage and V2G, connected to a real distribution network model. Fig. 2 shows the structure of the proposed case study implemented in the multi-site real-time co-simulation platform with decoupled subsystems. Through the VPN communication channel, the network model communicates the node voltages and a "control management signal" that represents the network availability/request for a power transfer with the distributed storage and V2G models. Based on these signals, considering the feedbacks provided by the distributed storage and V2G models (such as batteries state of charge, vehicle connection, etc.), the "management strategies" block properly operates the charge-discharge of the batteries to accomplish the local energy balance goal. In the following subsections, a brief description of the network, distributed storage and V2G models is reported.

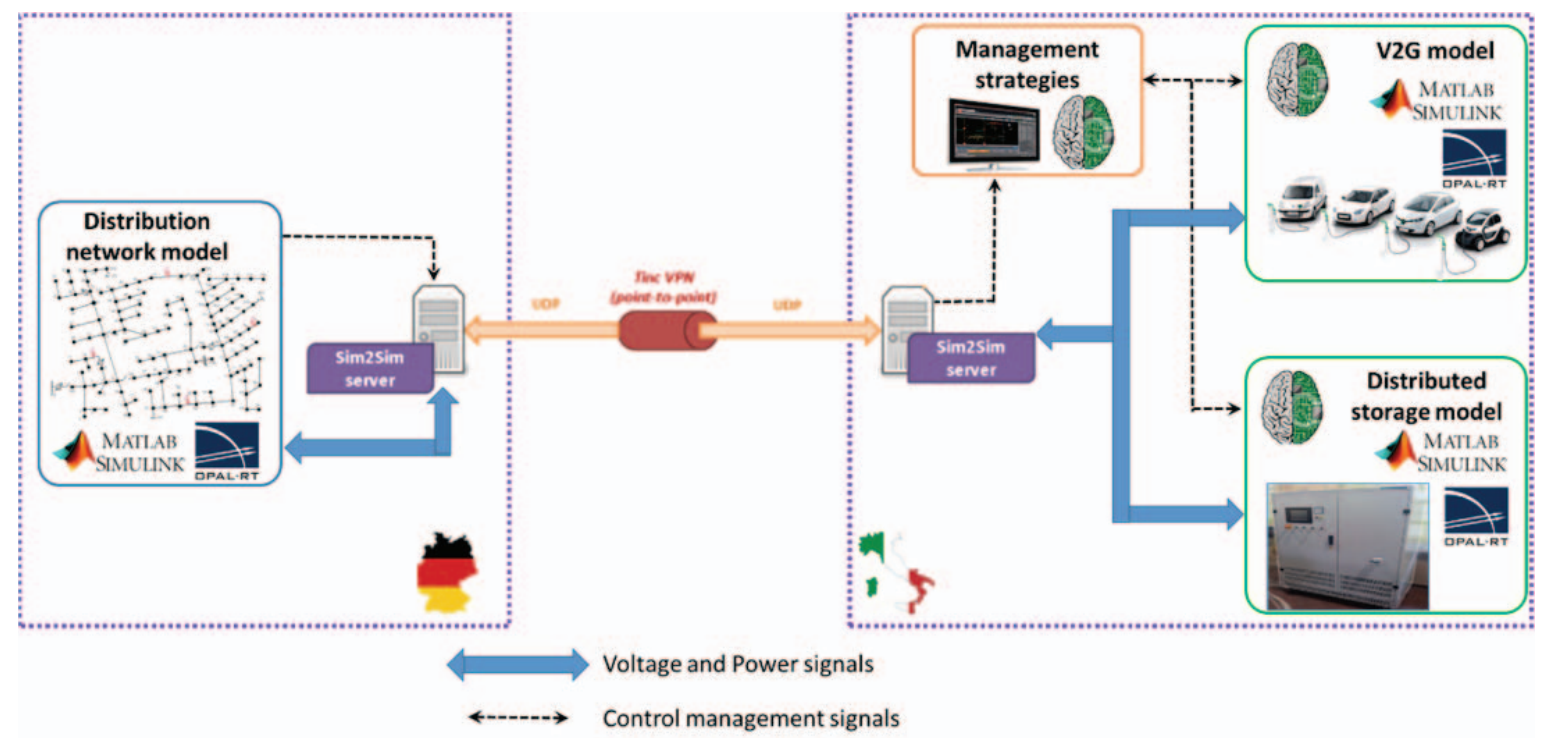

Fig.2. Structure of the proposed case study implemented in the multi-site real-time co-simulation platform.

\section{Network model}

A small portion of the real MV network of a city of around $800 \mathrm{k}$ inhabitants is modeled and the simulation runs on an Opal-RT target in RWTH Aachen. It consists of a primary substation with three MV-22kV busbars, each of which is fed by a transformer characterized by voltage ratio of $220 / 22 \mathrm{kV}$. Two transformers are characterized by a nominal power of 63MVA and the other one by a nominal power of 55MVA. For the case study, $1 \mathrm{MV}$ feeder derived from the HV/MV substation is selected, which is feeding $10 \mathrm{MV} / \mathrm{LV}$ substations. MV lines, mostly constituted by underground cables, connect the MV/LV substations. The feeder considered in the case study supplies loads for a peak consumption of about 5MW; at each substation both load and generation are connected. The power flow through the $\mathrm{HV} / \mathrm{MV}$ transformer measured in the primary substation, when no V2G and distributed storage are present, is similar to the one presented in Fig. 3. 
The model is built with the SimPowerSystem (SPS) toolbox of MatLab Simulink ${ }^{\circledR}$. The ARTEMiS software from OPAL-RT ${ }^{\circledR}$ is used to provide fixed-step solver dedicated to complex power systems. It is an add-in toolbox to SPS enabling hard real-time simulation of power systems. The main components of the distribution grid which are modeled for this case study are a three-phase voltage source in series with an RL branch as an equivalent model for the upstream HV grid connected to a swing bus, three-phase two-winding transformers, Artemis Stublines for decoupling the system and assigning the different portions to several computation cores, three-phase $p i$ section lines to model MV lines, and three-phase three-wire dynamic load models with external control of active and reactive power to model the prosumers.

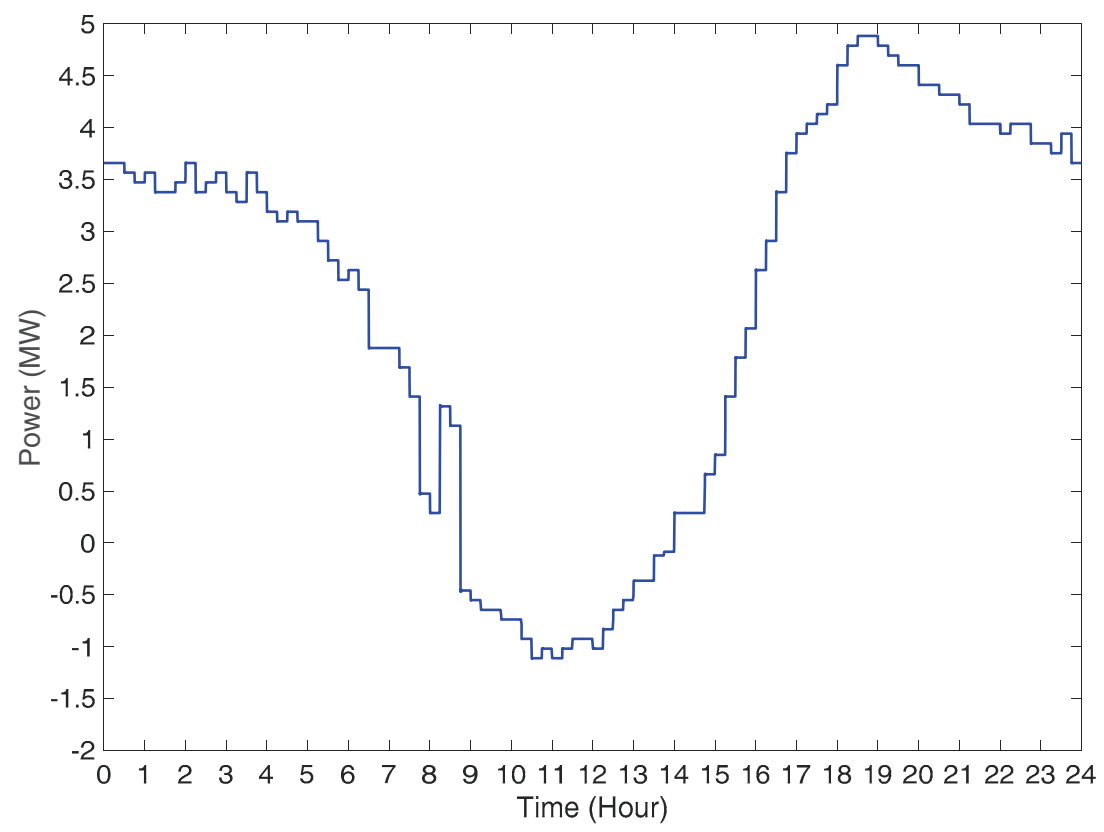

Fig. 3: Power flow in the HV/MV transformer without V2G.

\section{V2G and distributed storage model}

To evaluate the potential benefits in terms of local energy balance of the $\mathrm{V} 2 \mathrm{G}$ on a distribution network, it is mandatory to account for the energetic efficiency of the charge-discharge process of the on-board vehicular batteries.

The V2G concept necessarily involves the adoption of a vehicular battery charger based on bidirectional power electronics structure, such as active rectifiers. Fig. 4 shows the main components of a bidirectional battery charger suitable for distributed storage and electric vehicles equipped with V2G technology. The power electronics, composed by a bidirectional AC/DC and a bidirectional $\mathrm{DC} / \mathrm{DC}$ converter, is connected to the three-phase AC grid by means of a line side filter to limit the harmonic content of the absorbed current; for the same reason, a battery side filter is required between the $\mathrm{DC} / \mathrm{DC}$ converter and the battery.

In the charge-discharge process, the power converter and the battery are characterized by a different behavior in terms of energetic efficiency over the power range. In particular, the power converter is characterized by higher efficiencies in a power range close to its rated power, meanwhile, for the battery, low power ranges are typically preferred to increase the battery energetic efficiency and to limit the aging effect.

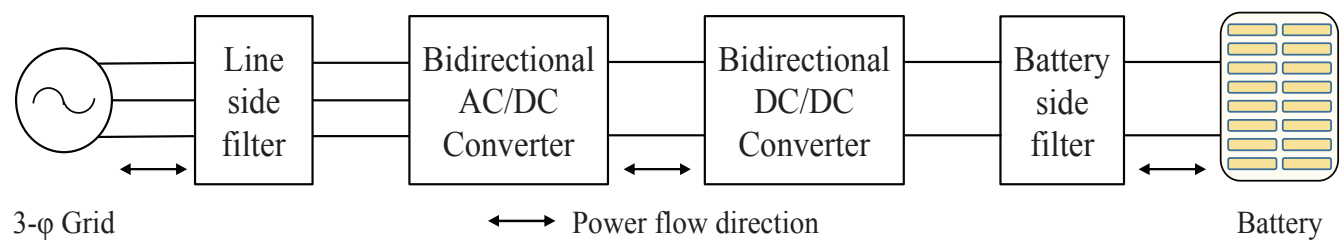

Fig.4. Main components of a bidirectional battery charger for electric vehicles and distributed storage. 
It is important to remark that a battery charger can be realized by means of different power converter architectures that can differ on the number of phases as well as voltage, current and power rates [13]. Similarly, different technologies and voltage, current and power levels are possible for the battery, depending on the required powertrain performance and storage capabilities.

In order to evaluate the efficiency of the power converter and the battery over a charge-discharge profile, different approaches can be used. Among them, those based on the circuit model require a detailed knowledge of each element composing the power converter and the battery systems; this information is often available to the producers only.

The V2G modelling approach proposed in this paper is based on efficiency maps for both the power converter and the battery. The efficiency maps can be gathered from technical literature, datasheets as well as experimental measurements conducted on real components. Fig. 5 shows a schematic of the V2G model implemented in Matlab/Simulink ${ }^{\circledR}$, highlighting both the input and the output signals. The modularity and scalability of the model allow simulating different power sizes; moreover, the efficiency maps can be of different complexity and resolution depending, case by case, on the information available for the systems.

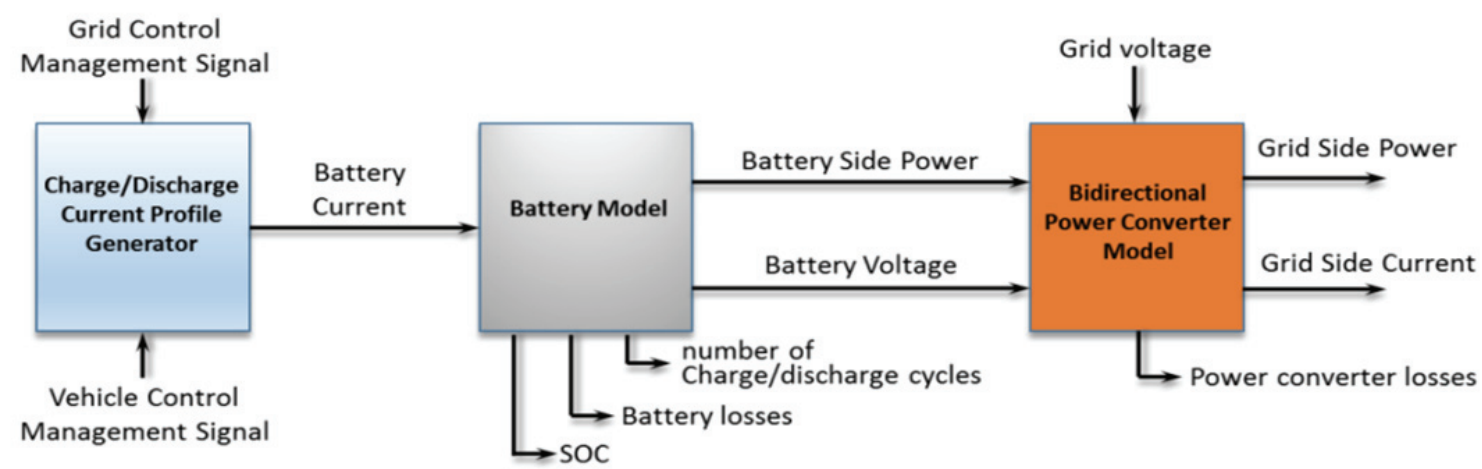

Fig.5. Schematic of the V2G model.

For the investigation proposed in this paper, the efficiency map for the power converter has been obtained by laboratory measurements conducted for a $14 \mathrm{~kW}$ bidirectional power converter; the tested structure is representative of a battery charger for vehicles equipped with V2G technology. The test procedure that has been conducted for the efficiency measurements can be applied to any battery charger topology. In particular, the measurements have been done for a grid voltage equal to $400 \mathrm{~V}$ and different battery voltage levels ranging from $350 \mathrm{~V}$ down to $100 \mathrm{~V}$ with a step of $25 \mathrm{~V}$. For each value of battery voltage, the battery and the grid side voltages, currents and powers have been measured for different battery currents in the range $+40 \mathrm{~A}$ to $-40 \mathrm{~A}$ with a step of $5 \mathrm{~A}$. Fig. 6(a) shows the efficiency curves measured on the prototypal power converter both in charge and in discharge; the same results are shown in the 3D efficiency map presented in Fig. 6(b). The measurement results show that for the tested power converter higher efficiencies are obtained during the discharge process.

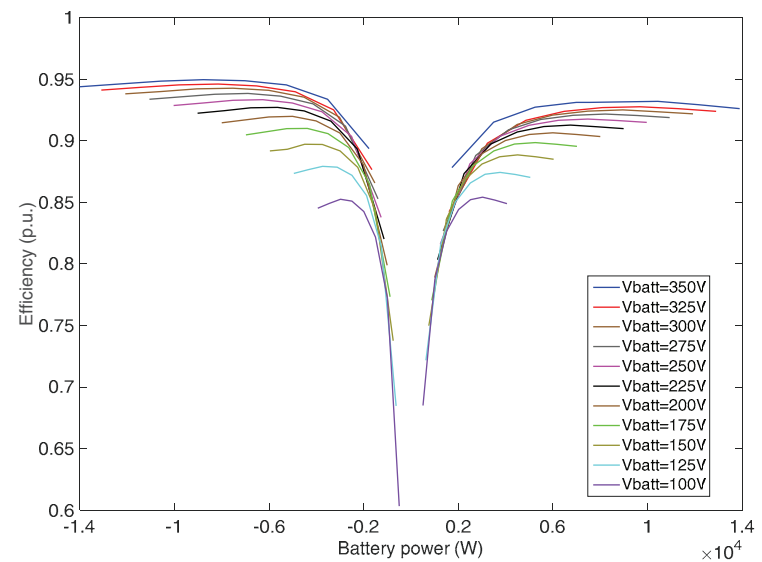

(a)

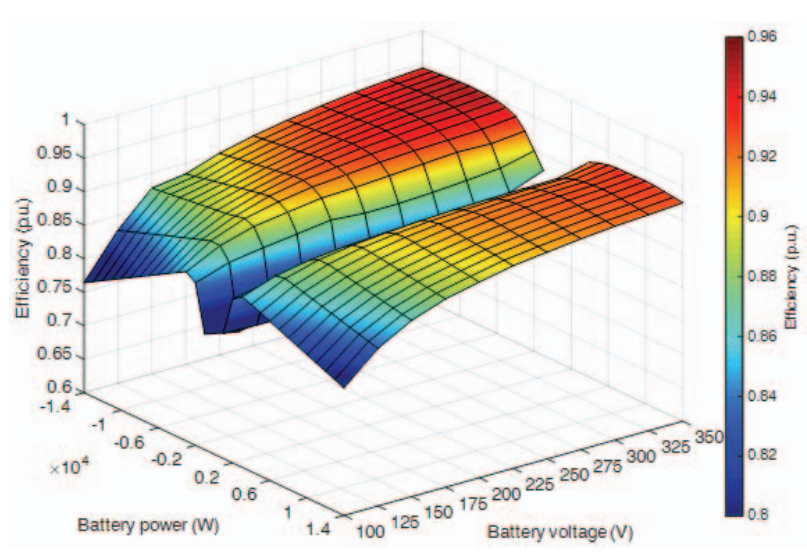

(b)

Fig.6. Efficiency map measured for a prototypal bidirectional power converter. 
Fig. 7 shows the prototypal $14 \mathrm{~kW}$ bidirectional power converter and the test bench setup for the efficiency measurement.

It has to be remarked that the model of stationary distributed storage systems can be simulated using the same model developed for the V2G. Obviously different voltage, current and power levels have to be considered with respect to a vehicle, and this is possible thanks to the modularity and scalability of the developed model.

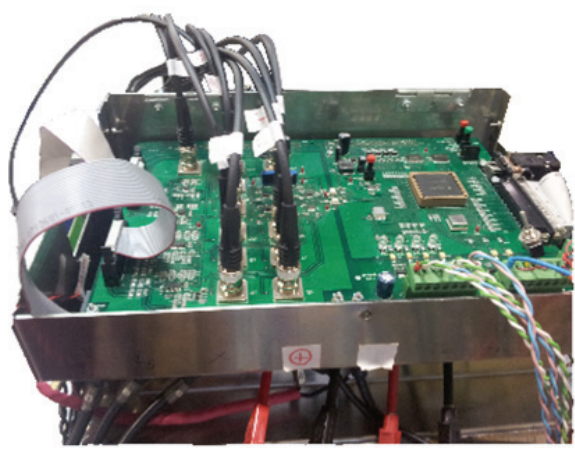

(a)

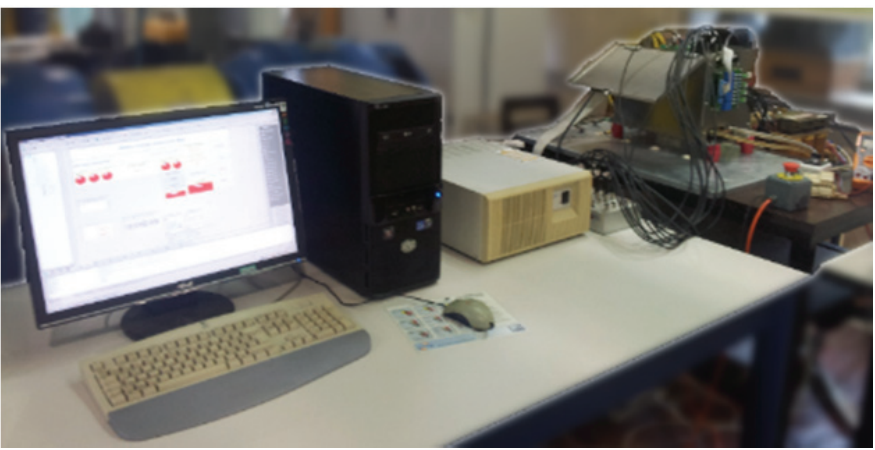

(b)

Fig.7. Prototypal bidirectional power converter: (a) detail of the power electronics control signals communication interface; (b) test bench setup.

\section{Simulations and results}

Based on the case study previously presented, to assess the potentialities of the developed cosimulation platform, two scenarios have been simulated and compared. In particular, in the first scenario, 120 vehicles are connected to the modelled distribution network concentrated in a single MV/LV substation, as sketched in Fig. 8(a). In the second scenario the vehicles have instead been subdivided into three parking lots connected at three different MV/LV substations: 40 vehicles are connected at substation 1, while 30 and 50 are connected at substations 2 and 3, respectively, Fig. 8(b).

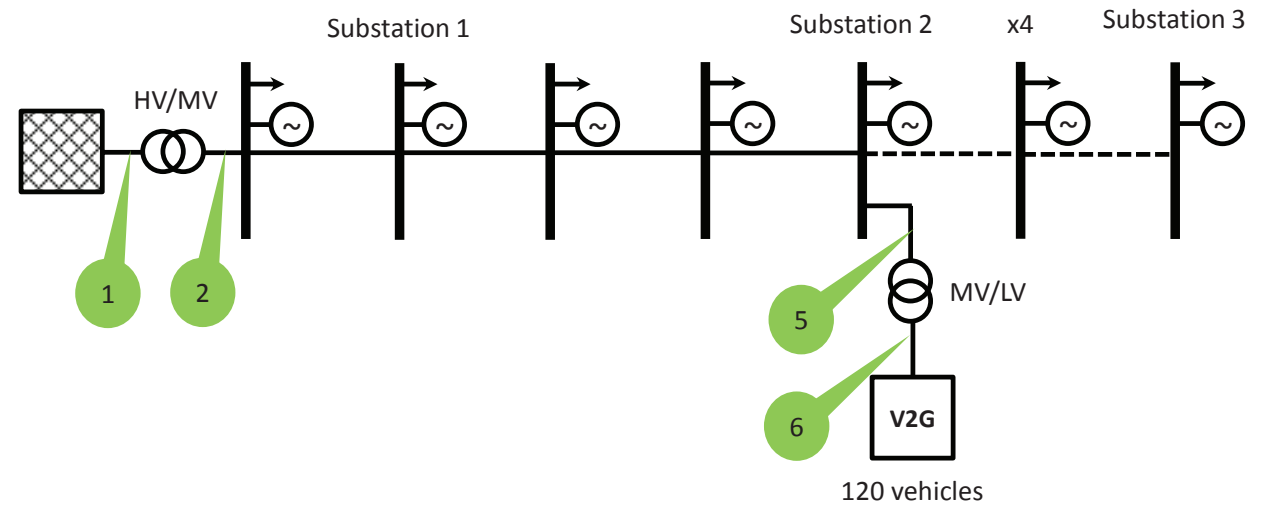

(a)

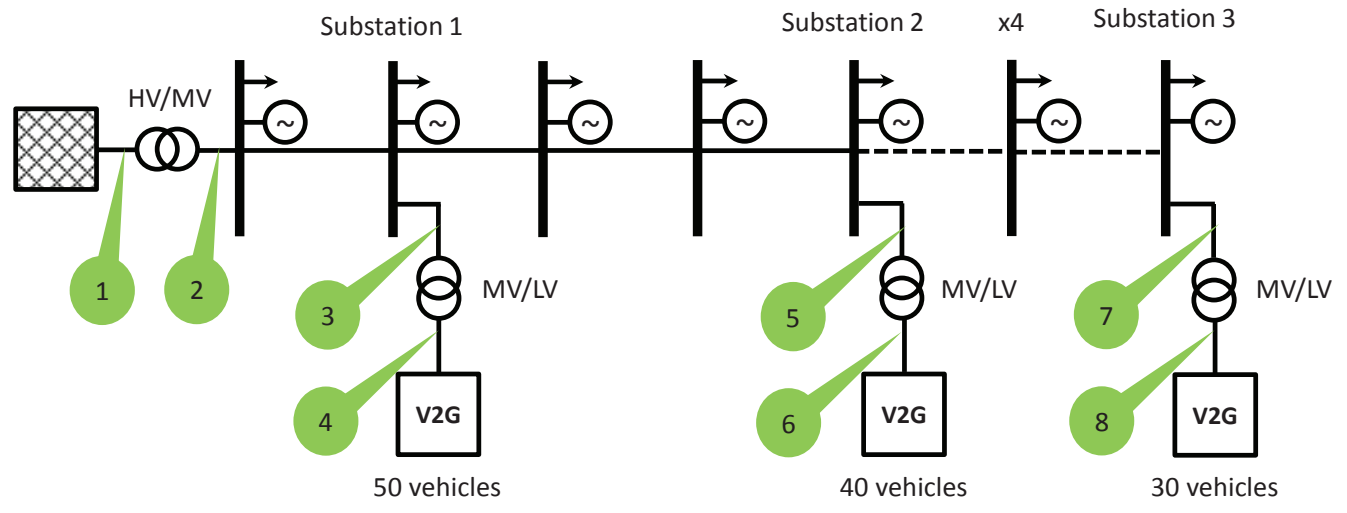

(b)

Fig.8. Simulated feeder with V2G connection and measurement points: (a) concentrated scenario;

(b) distributed scenario. 
Both scenarios have been simulated considering a specific management strategy to achieve local energy balance in order to reduce the power flow through the HV/MV transformer. For this purpose, the power flow through the HV/MV transformer (measurement point 2 in Fig. 8) is compared with 2 thresholds $(0$ and $3 \mathrm{MW})$ : when the power absorbed from the HV grid is higher than $3 \mathrm{MW}$ the request is for battery discharge, vice versa, when the power flow is negative, the request is for battery charging. To avoid oscillations, two different thresholds have been set for the activation and deactivation signals. As an example, Fig. 9(a) presents the power absorbed in the primary substation with distributed V2G, together with activation (solid lines) and deactivation (dashed lines) thresholds. It is important to highlight that the charge or discharge of the vehicular batteries depends also on their SOC, which is allowed to range between $40 \%$ and $90 \%$ to guarantee a minimum stored energy for the user and to limit the battery aging effect. Fig. 9(b) presents the batteries SOC and the charge/discharge logic signal. The former is expressed in percent, while the latter assumes the values +1 or -1 when charging or discharging are requested, respectively. When the logic signal is 0 no charging or discharging is requested by the management system. In the simulation the batteries initial SOC is $40 \%$ and for this reason, even if the logic signal is -1 (discharge), in the first 6 hours no energy is provided by the batteries. Slightly before the $9^{\text {th }}$ hour the lower threshold activates the charging, that is then stopped when the SOC reaches the maximum allowed value of $90 \%$.

Comparing the absorbed power without and with V2G (Fig. 3 and Fig. 9(a), respectively), it is well evident the levelling effect, with a reduction of both the peak and the injected power.

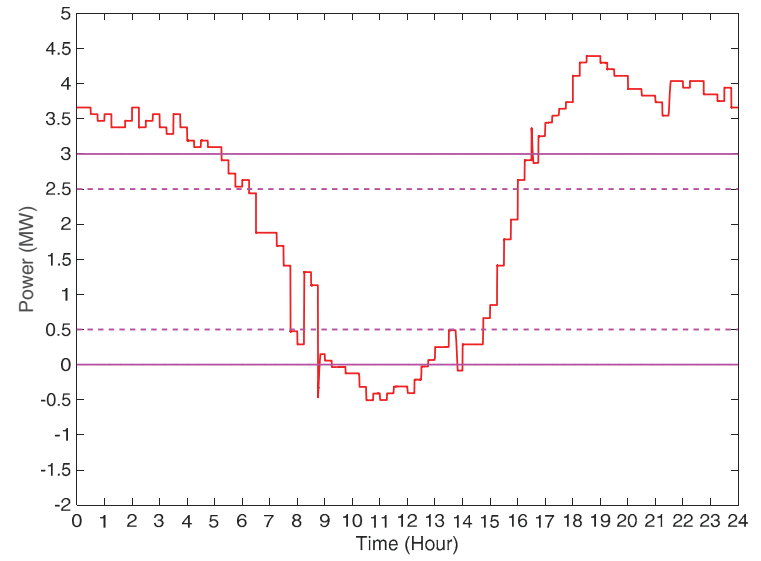

(a)

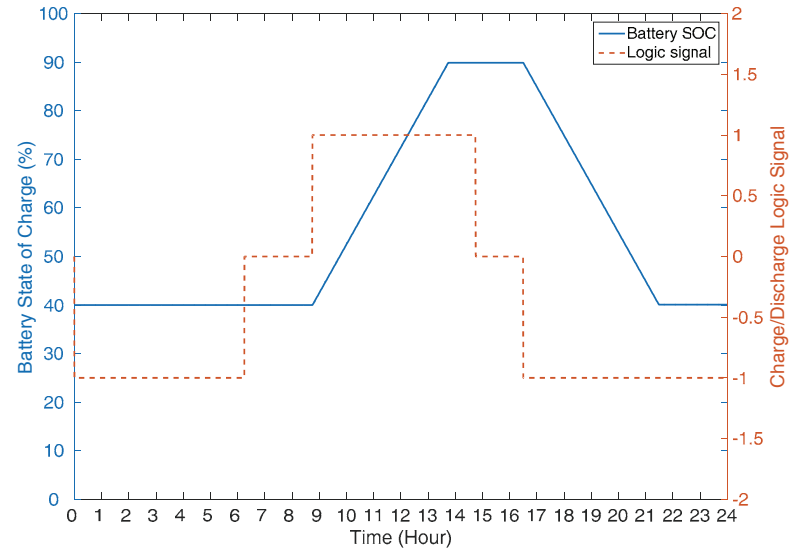

(b)

Fig. 9: (a) power flow in the HV/MV transformer with V2G; (b) batteries SOC and charge/discharge logic signal.

Fig. 10(a) shows the power absorbed and injected by the vehicles when they are concentrated in one substation. Fig. 10(b) refers to the second scenario, where the vehicles are distributed in three different substations. In both figures, solid lines correspond to the power measured at the MV side of the $\mathrm{MV} / \mathrm{LV}$ transformer, while dashed lines correspond to the LV measurements. The measurements points are highlighted in the feeder sketched in Fig. 8.

The power flow in the HV/MV substation (measurement point 2 in Fig. 8) is sent using the VPN (through UDP blocks and Sim2Sim interface) from the network model to the V2G model for the comparison with the activation and deactivation thresholds. The voltages measured at the V2G connection points (measurement points 4, 6 and 8 in Fig. 8) are also sent to the V2G models to be considered for the efficiency of the power converter and battery. Vice versa the powers absorbed or injected by V2G systems, calculated by the vehicles models, are sent from the real-time simulator in Turin to the one in Aachen, where controllable loads (modeled as three-phase three-wire dynamic loads with external control of active and reactive power) absorb or inject the power from or into the network.

The communication delay of this multisite real time co-simulation is about $13 \mathrm{~ms}$, which is shorter than the loads profiles step length. In addition, Sim2Sim servers drop reordered and invalid packets ensuring that delay variations do not introduce surplus dynamic. 


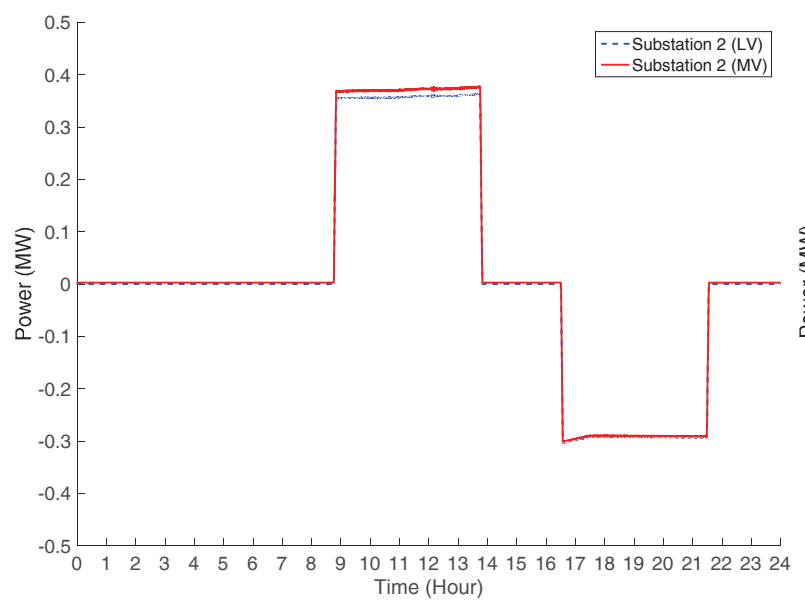

(a)

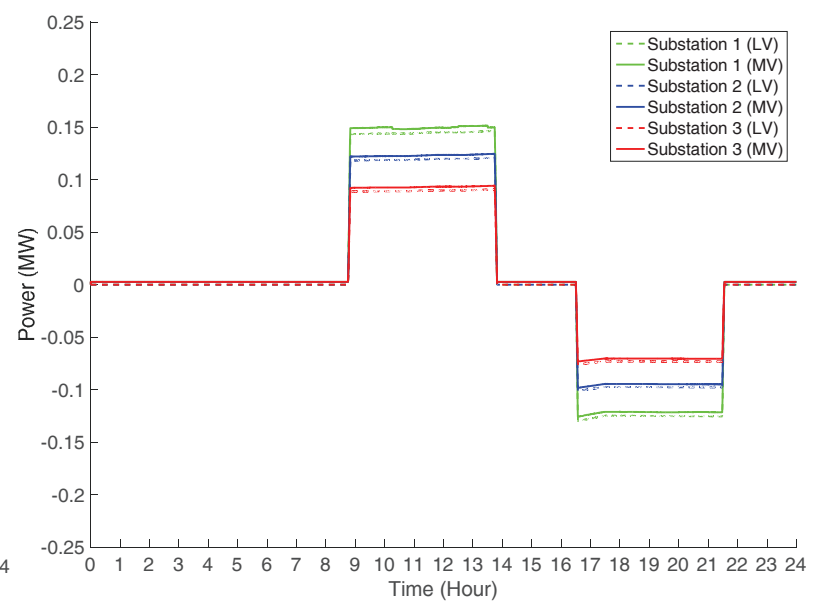

(b)

Fig. 10: Vehicles absorbed/injected power: (a) all vehicles concentrated in substation 2; (b) vehicles distributed in 3 different substations.

Table I summarizes the net energy absorbed from the HV network and the losses of the different components in the two simulated scenarios. It is well evident that changes in the storage and V2G connection to the grid impact the system performance. It has to be pointed out that the results here reported have been obtained applying a basic control strategy; however, also different and more sophisticated management strategies could be applied and tested thanks to the developed simulation framework. In addition, as previously mentioned, it would be possible to test different battery and power converter models and, in general, any prosumer model which could be connected to the distribution grid.

Table I: Simulation results

\begin{tabular}{|l|c|c|c|}
\hline & $\begin{array}{c}\text { V2G concentrated in } \\
\text { substation 2, MWh }\end{array}$ & $\begin{array}{c}\text { V2G distributed } \\
\text { in 3 different } \\
\text { substations, MWh }\end{array}$ & $\begin{array}{c}\text { Percentage } \\
\text { variation }\end{array}$ \\
\hline $\begin{array}{l}\text { Net energy absorbed } \\
\text { from the HV network }\end{array}$ & 55.58 & 55.64 & $+0.1 \%$ \\
\hline MV lines losses & 0.36 & 0.34 & $-6.5 \%$ \\
\hline HV/MV transformer losses & 1.02 & 1.02 & $0 \%$ \\
\hline MV/LV transformers losses & 0.12 & 0.22 & $+87 \%$ \\
\hline Vehicles losses & 0.32 & 0.28 & $-12 \%$ \\
\hline
\end{tabular}

\section{Conclusion}

In this paper a multi-site real-time co-simulation platform for studying the interactions of hardware and software components with the distribution network has been presented. The possibility of simulating different subsystems at different laboratories can solve the problems related to confidentiality concerns of software and hardware manufacturers, as well as research institutions and network operators. In this paper a case study regarding the integration of distributed storage and V2G systems has been presented. The model of a distribution network, running on a real-time simulator in Aachen, has been interconnected to the model of $\mathrm{V} 2 \mathrm{G}$ and distributed storage, running on another real-time simulator, in Torino. The two models are interacting in a co-simulation, exchanging limited data, and in particular the power flowing in the HV/MV transformer, the bus voltages and the powers absorbed or injected by V2G systems. 
The assessment has been conducted testing a basic management strategy for different configurations of V2G connected to a distribution network. The simulations conducted on the case study show that the delay introduced by the communication between the real time simulators does not affect the simulation results. The implemented co-simulation is applicable and beneficial for all cases in which different parties would perform tests maintaining the confidentiality on their products and algorithms.

\section{Acknowledgements}

The work presented in this paper has been performed in the framework of the Eric-lab initiative (http://www.eric-lab.eu/).

\section{References}

[1] E. Ragazzi, E. Bompard, A. Tenconi, "Electric Vehicles and Power Grids: Challenges and Opportunities", The Greening of the Automotive Industry, Editore: Palgrave Macmillan Ltd., Basingstoke (GBR), ISBN 978-0-2303-6909-2.

[2] H. Farhangi, "The path of the smart grid", Power and Energy Magazine, IEEE, vol. 8, no. 1, pp. 18-28, 2010.

[3] X. Yu, C. Cecati, T. Dillon, M.G. Simões, "The New Frontier of Smart Grids", IEEE Ind. Electron. Magazine, vol.5, no.3, 2011, pp.49-63.

[4] C. Liu, K.T. Chau, D. Wu, S. Gao, "Opportunities and Challenges of Vehicle-to-Home, Vehicle-to-Vehicle, and Vehicle-to-Grid Technologies", Proceedings of the IEEE, vol.101, no.11, November 2013, pp. 24092427.

[5] NIST Framework and Roadmap for Smart Grid Interoperability Standards. NIST National Institute of Standards and Technology.

[6] C. Dufour, S. Araujo, and J. Belanger, "A survey of smart Grid research and development involving realtime simulation technology," in Innovative Smart Grid Technologies Latin America (ISGT LA), 2013 IEEE PES Conference On, 2013, pp. 1-8.

[7] S. S. Biswas, F. Shariatzadeh, R. Beckstrom, and A. K. Srivastava, "Real time testing and validation of Smart Grid devices and algorithms," in Power and Energy Society General Meeting (PES), 2013 IEEE, 2013, pp. 1-5.

[8] M. Stevic, S. Vogel, A. Monti, S. D'Arco, "Feasibility of geographically distributed real-time simulation of HVDC system interconnected with AC networks," in PowerTech, 2015 IEEE Eindhoven, June 2015.

[9] M. Stevic, A. Benigni, A. Monti, "Development of a simulator-to-simulator interface for geographically distributed simulation of power systems in real time," in Industrial Electronics Society, IECON 2015 - 41st Annual Conference of the IEEE.

[10] J. Verseille, K. Staschus, "The Mesh-Up: ENTSO-E and European TSO Cooperation in Operations, Planning, and R\&D", in Power and Energy Magazine, IEEE , vol.13, no.1, pp.20-29, Jan.-Feb. 2015.

[11] A. Estebsari, E. Pons, E. Patti, M. Mengistu, E. Bompard, A. Bahmanyar, S. Jamali, “An IoT Realization in an Interdepartmental Real Time Simulation Lab for Distribution System Control and Management Studies", in 2016 IEEE $16^{\text {th }}$ International Conference on Environment and Electrical Engineering (EEEIC), June 2016.

[12] Tinc VPN Project Home Page. [Online]. Available: http://tinc-vpn.org. [Accessed: 08.11.2015]

[13] M. Yilmaz, P.T. Krein, "Review of Battery Charger Topologies, Charging Power Levels, and Infrastructure for Plug-In Electric and Hybrid Vehicles", IEEE Trans. on Power Electron., vol.28, no.5, May 2013, pp. 2151-2169. 